\section{Birkbeck Library: the impact of an online induction tutorial}

\author{
Martin Draper
}

be abandoned as we were defeated by the short timescale available in trying to achieve a complex set of partnerships as well as overcoming some bureaucratic barriers we had not anticipated.

The second topic focus was the impact of a newly developed online induction tutorial which was being deployed in September 2003 for the first time. The tutorial was branded as LIFE, Library Induction for Everyone, and had been developed in-house by library staff and the College Web Development Team. Given the time lost, this topic appealed because it was fully within our control and was related to the theme of the original design.

\section{Importance of the topic for Birkbeck Library}

Whilst the refocusing on the LIFE induction tutorial was to some extent opportunistic and driven by a need to catch-up, it nevertheless addressed a topic of great importance to the Library. The tutorial had been developed as part of a potential answer to a pressing need. Face to face induction sessions were consuming an ever-increasing proportion of the Subject Librarians time yet a significant proportion of new students, for various reasons, still had no access to them. We were concerned to widen this access by changing our methods of induction but were also concerned that such changes should not impair the quality of induction provided. Consequently, we needed to try and measure the qualitative impact of our tutorial.

\section{Application of the Impact Implementation Methodology}

What the service was trying to achieve in the area that is being investigated In terms of widening access to induction, LIFE is a web-based online tutorial and therefore available for access at all hours, from any internet-connected computer, allowing repeated consultation as and when required. We also wanted it however, to act as a catalyst for change in the way we structured library 
induction as our established practices were unsustainable. There was a strong sense too that the tutorial should contribute towards, and reinforce, fledgling eLearning initiatives within the College, thereby demonstrating library support for them. Ideally, its implementation was to be followed by the creation of subject specific information skills modules built within WebCT, the VLE utilised at Birkbeck. In order to do this we would require extra support from the College so LIFE needed to have a strong and positive impact politically.

\section{Success criteria}

Together these considerations strongly influenced the success criteria we identified for the topic. We were looking for a large increase in the number of students receiving induction. We were seeking an increase in levels of student awareness of library resources and services and an improved level of understanding of search strategy techniques. We wanted course designers using WebCT to adopt the tutorial and incorporate it within their designs. We also wanted to receive subsequent requests to either attend information skills sessions or for us to develop subject-based information skills tutorials.

\section{Impact measures identified}

Partly due to restricted time we decided to focus on a small but representative group of students who had had no prior exposure to the tutorial nor benefited from a formal induction session. We sought to collect data and observe practice amongst this group. In addition, we would examine existing data collection and established feedback, comments and complaints mechanisms for hints of impact outside the sample group.

\section{Evidence collected}

An attempt was made to analyse the library use, knowledge, and "behaviour" of each test group member prior to tutorial exposure. For example, they had to record their search strategies for defined tasks and we examined records to see whether they had registered for personal Athens accounts. All the areas examined were re-examined in the months following exposure to LIFE and, where existing comment, survey and feedback mechanisms proved useful they were monitored continually.

\section{Methods used}

A student test group was recruited and participated in a formal, supervised, session in November 2003. Each student was asked to complete a pre-questionnaire before then undertaking the LIFE tutorial (they needed to complete the full tutorial as they were also being asked to give feedback on tutorial content and design).

The pre-questionnaire had three sections which sought information regarding $(a)$ their current use of library services, $(b)$ their existing knowledge of the library (together with its policies and services), and (c) their practices when searching for information.

In April 2004 (five months later) each member of the test group was sent the same questionnaire to complete once more, this time in an uncontrolled setting. The sections and questions were identical to the prequestionnaire. Each respondent had of course been exposed to the tutorial since completing the pre-questionnaire and we were interested in examining whether there were significant differences in their responses.

Monitoring of existing response and request mechanisms has been mentioned. We also, perhaps habitually, resorted to the quantitative technique of collecting induction session attendance figures and examining tutorial access $\log$ data.

\section{Particular research instruments of interest}

In retrospect it is clear that we relied over heavily on the survey questionnaire as our research instrument. We worked hard in constructing a test group that was fairly representative of the College student membership and then maintaining contact with that group. The response rate we received for 
the second questionnaire was initially just below $50 \%$ which confirms a frequent criticism of distributed surveys. However, using address and course contact information we repeatedly chased the non-responses and ultimately achieved a healthy response rate of $90 \%$. The questionnaire allowed us to quickly collect information regarding student usage and knowledge of services and provide a comparison at a later stage. What it was unable to do, however, was to meaningfully identify or comment upon student design of search strategies and techniques.

\section{How the results have been used}

Members of the test group performed significantly better in the post-questionnaire than they had in the pre-questionnaire for sections $(a)$ and $(b)$ but failed to show consistent improvement in section $(c)$. Thus their use and knowledge of the library resources and services had improved markedly over the intervening five months and the tutorial is seen as a factor contributing to this. The lack of improvement in the tests regarding search strategies could be seen as highlighting the differences between induction and information skills teaching. It could be seen as underlining the need to create online information skills tutorials in order to crucially enhance and build upon the induction experience. It perhaps challenges the assumptions behind our seeking to observe improved search behaviour as a consequence of exposure to a library induction tool. Unfortunately, we believe, it also reflects our inappropriate use of the questionnaire alone to test this particular area.

Student access to induction was widened. For the 2003/04 academic session our Subject Librarian team continued with the face-to-face tutorial structure as before despite the creaking structure. LIFE was publicised both at these sessions and more widely but it was not used as the centre-piece at formal induction as had been intended. As in the past the number of students attending these sessions increased. In addition to these numbers was the large number of accesses the tutorial received. It is not possible to be certain who was accessing the tutorial or for what reason via the log statistics but the access figures from within the Birkbeck domain alone remained high throughout the three academic terms. Also of interest was the average length of tutorial visit recorded: twelve minutes in the autumn term climbing to just over twenty minutes for both the spring and summer terms. This suggests to us that a different use was made of the tutorial once familiarity and orientation had been achieved in term one, a use perhaps pointing to the need for information skills tutorials.

The Subject Librarians voluntarily redesigned the induction process for the autumn term of the 2004/05 academic year. For the first time they decided to abandon the face-to-face sessions in favour of a rolling programme of library tours open to all during which the tutorial was both advertised and briefly demonstrated. This partly reflected their confidence in the tutorial which had not been present the year before and we view it as an indication of successful tutorial impact.

The tutorial was adopted by the College VLE administrative team and placed as a bookmark within all WebCT accounts. Additionally, the Library was invited to bid for college development funding to create six subject-based information skills tutorials to be embedded within WebCT delivered courses. This bid for an AfterLIFE project is still being assessed.

\section{What was learnt from participation?}

Participation within the project caused us to challenge many of our assumptions concerning the way we collect data and use it as a measure of performance or impact. This is the start of an ongoing and radical process.

We are not entirely happy with our first attempt to implement these ideas. Whilst we learnt a lot about survey design and the formation and organisation of subject test groups we feel we should have used, or experimented with, a wider range of tools and methods. The survey, for example, should have been supported by focused interviews and observed student 
searching. This may have allowed us to discover something more meaningful regarding their search behaviour and facilitated a greater dialogue between the group subjects and ourselves. We have begun to appreciate that all research tools and techniques have strengths and weaknesses and that there is unlikely to be a single tool or method that will readily help to answer the impact question by itself. A greater familiarity with these tools is required before we can confidently deploy them in an appropriate and effective way. This is one of the challenges that IMPACT involvement has set us. 